\title{
Knowledge Awareness and Attitude About Needle Stick Injury Among Dental Practitioners in Chennai
}

\author{
Sharmila ${ }^{1}$, M.P Brundha ${ }^{2 *}$ and V.B. Preejitha ${ }^{3}$ \\ ${ }^{1}$ Saveetha Dental College and Hospitals, Saveetha Institute of Medical Science \\ and Technical Sciences, Saveetha University, Chennai -77,Tamil Nadu, India \\ ${ }^{2}$ Associate Professor, Department of Pathology, Saveetha Dental College and Hospitals, \\ Saveetha Institute of Medical Science and Technical Sciences, Saveetha University, \\ Chennai-77,Tamil Nadu, India \\ ${ }^{3}$ Tutor Department of pathology Saveetha Dental College and Hospitals, Saveetha Institute of Medical \\ Science and Technical Sciences, Saveetha University,Chennai -77,Tamil Nadu, India
}

\section{ABSTRACT}

Needle-stick injury (NSI) is one of the maximum potential risks for health care people. They pose a substantial risk of occupational transmission of blood-borne pathogens. Many numbers of health care people remain susceptible to deadly viruses especially, bloodborne pathogens. Dental professionals are more prone to Needlestick injury due to their limited working area. The main cause of this injury is due to improper capping of the needle, a technique used, handling the specimens, etc. The present study was done to determine knowledge and attitude about needle stick injury among dental practitioners and the action taken by them after the incident. A self-designated 15 questions were created and it was circulated among 100 dental practitioners. The results were collected and analyzed using SPSS. An effective plan should be made for the prevention and management of injury especially among health care professionals. Among 100 responses most of the people do not follow the protocol which indicates that there is a lack of adequate knowledge. From this study, we conclude that only 50\% of the study population were aware about the needle stick injury and the infections spread by Needle stick injury. Even though a majority of the females knows about the Hepatitis B vaccination and its relation to needle stick injury when compared to males but they didn’t undergo vaccination properly. There is a precise scope of development in terms of reporting and prevention of needlestick accidents.A need for awareness programs among dental practitioners repeatedly has been raised

KEY WORDS: NEEDLESTICK INJURY, BLOODBORNE PATHOGENS, AWARENESS PROGRAMS, HEALTH CARE PROFESSIONALS.

\section{ARTICLE INFORMATION}

*Corresponding Author: brundha.sdc@saveetha.com

Received 18th June 2020 Accepted after revision 5th August 2020

Print ISSN: 0974-6455 Online ISSN: 2321-4007 CODEN: BBRCBA

Thomson Reuters ISI Web of Science Clarivate Analytics USA and Crossref Indexed Journal

$$
\begin{aligned}
& \text { Clarivate } \\
& \text { Analytics }
\end{aligned}
$$

NAAS Journal Score 2020 (4.31) SJIF: 2020 (7.728)

A Society of Science and Nature Publication,

Bhopal India 2020. All rights reserved.

Online Contents Available at: http//www.bbrc.in/

Doi: http://dx.doi.org/10.21786/bbrc/13.7/95 


\section{INTRODUCTION}

Needlestick damage (NSI) is a penetrating stab wound from a needle (or another sharp object) which may additionally result in blood-borne infections which include AIDS, Hepatitis B, and Hepatitis C(Kapila, Gupta and Chopra, 2008). The common risk for transmission after percutaneous exposure is about $0.3 \%, 6-30 \%$, and $1.8 \%$ for human immunodeficiency virus (HIV), hepatitis $\mathrm{B}$, and hepatitis $\mathrm{C}$, respectively. Over $90 \%$ of those infections arise in low-income nations and maximum are preventable(Walley, 2014).

Needlestick accidents can occur for the duration of utilization or disposal of sharps(Alrumayyan. et al., 2018). It is crucial to dispose of the sharps well, in any other case they could become involved in linen or garbage and may injure the medical examiners who come upon them unexpectedly(Agarwal, Wakhlu and Srivastava, 2016). Though many articles have been published about the prevention of this injury significant success has not been achieved (Nanda et al., 2019).

A needle stick injury is caused due to penetration by a needle or any other sharp object and it leads to transmission of bloodborne diseases, (Walley, 2014) placing those exposed at increased risk of contracting infectious diseases, such as hepatitis B (HBV), hepatitis $\mathrm{C}$ (HCV), and the human immunodeficiency virus (HIV) (Mahajan and Gupta, 2019). Among healthcare workers and laboratory personnel worldwide, more than 25 blood-borne virus infections have been reported to have been caused by needlestick injuries (Agarwal, Wakhlu and Srivastava, 2016).In addition to needlestick injuries, the transmission of these viruses can also occur as a result of contamination of the mucous membranes, such as those of the eyes, with blood or body fluids, but needlestick injuries make up more than $80 \%$ of all percutaneous exposure incidents in the United States (Mahajan and Gupta, 2019). Various other occupations are also at increased risk of needlestick injury, including law enforcement, laborers, tattoo artists, food preparers, and agricultural workers(Ayas et al., 2006).

The routine use of sharp instruments in dental remedy, the presence of blood and saliva, and the various bacterial vegetation inside the oral hollow space all contribute to the dangerous nature of the dental administrative center for blood-borne infections (Ayas et al., 2006; Singh et al., 2017). Preventing NSIs is an undertaking confronted in actually each medical work vicinity. In a dental environment, the burden of NSIs and SIs can be decreased when a dental professional abides through the cutting-edge and universally typical precautionary measures against NSIs(Ayas et al., 2006). Every healthcare facility ought to have an infection control application in place via a working sanatorium contamination control committee(Olubuyide and Olawuyi, 1995)

This study has been conducted to assess the knowledge, attitude, and awareness of needle stick injury among dental practitioners. The main objectives of this study were, To investigate and evaluate the extent of attention, mindset, and practices regarding Needle Stick Injury, standard precautions and injection practice among dental practitioners, To study the factors resulting Needle Stick Injury and to assess hepatitis B immunization status among the practitioners(Dafaalla, 2016).

\section{MATERIAL AND METHODS}

Sample collection: A survey was conducted among 100 dental practitioners of Chennai in the month of November 2019. Nearly 15 valid questions had been prepared and circulated among the dental practitioners and answers were recorded.

Inclusion Criteria: Selection criteria include all the dental practitioners of Chennai who are willing to participate in the study.

Exclusion Criteria: Dental practitioners who were not willing to participate were excluded from this study. Undergraduate and Postgraduate dental students are excluded from this study.

Sampling method: In the present study,the sampling method used is the Random sampling method.

Data Collection and Tabulation: The responses were entered into the excel sheets and then tabulation of the data finally and the question comparison was done. The representation of the data is through the bar graph.

Statistical Analysis: The statistical software used IBM SPSS V22.The statistical tests used were descriptive analysis and Chi Square analysis. Significant $\mathrm{p}$ value was set at $<0.05$.

\section{RESULTS AND DISCUSSION}

Dental health care employees are uncovered to bloodborne virus infections of their professionals as they are in near contact with the infected person's saliva and blood. These viruses are transmitted through NSIs. There has been various research carried out in India and across the world which displays that a huge proportion of dental practitioners are vulnerable to these viral infections(Wick, 2001).

A kind of dental strategy conveys the danger of NSIs including administration of anesthesia, intermaxillary fixation, suturing, root canal treatment, manipulation of sharps, and extractions(Thomas, 2020). Specialists told that suturing is the main cause of NSI because it aids with human extraction which is also a common infection (Alrumayyan. et al., 2018; Thomas, 2020). This is explained with the aid of the fact that students are inexperienced and there is a lack of information in following common precautions protocol by college students.

In the present study, $40 \%$ of the respondents were males and $60 \%$ of the respondents were females (Figure 1).In 
the present study, 56\% percent had been aware of the needle stick harm and the remaining $44 \%$ of people were not aware of needle stick injury (Figure 2)Therefore, the information about needle stick injury among dental practitioners changed into insufficient.

Figure 1: Pie chart represents that $60 \%$ are females (green) and $40 \%$ are males (cream) participated in the survey.

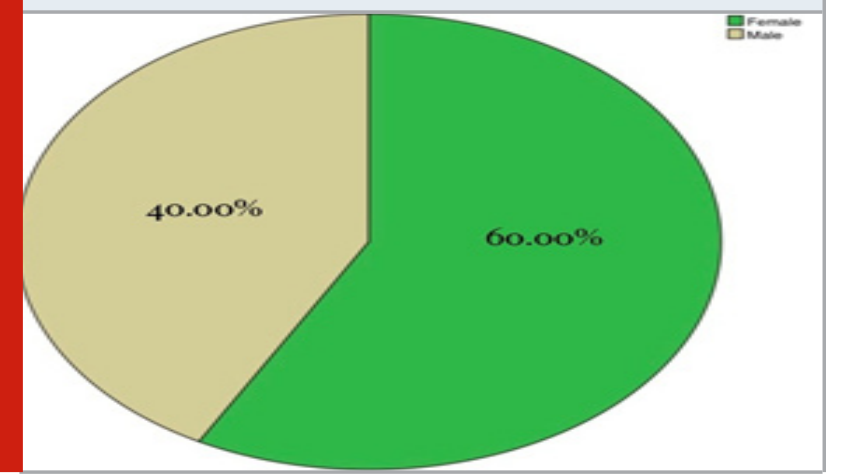

Figure 2 : Pie chart represents that $56 \%$ of the participants have been exposed to needle stick injury (cream) and $44 \%$ of the participants have not been exposed to injury ( green).

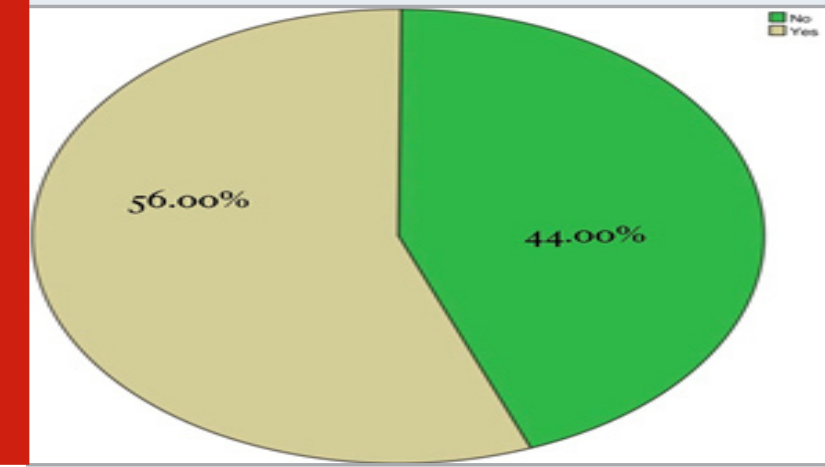

Figure 3: Pie chart represents that $44 \%$ of the participants squeeze their finger immediately after the injury (cream) and $56 \%$ of the people do not squeeze their finger (green).

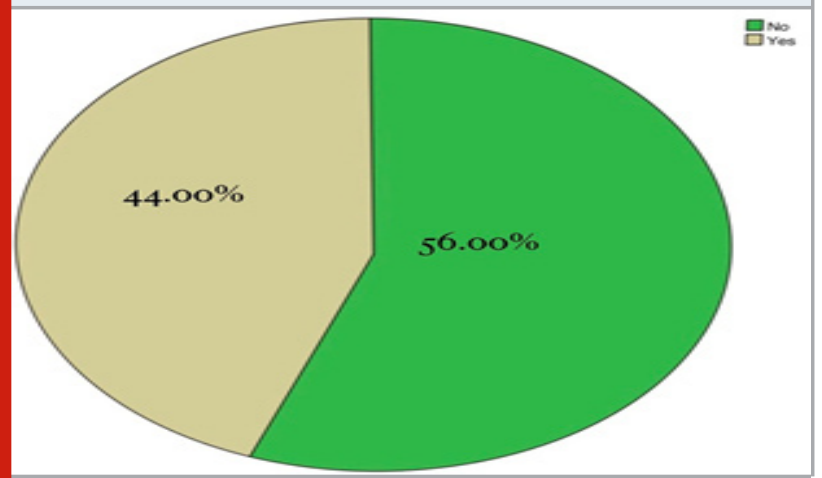

When the reporting status of HCWs was assessed, it was found that almost 54\%of the people did not report NSI and $46 \%$ of people reported the incident (Figure 5). The present study shows that 56\% of the people do not squeeze their fingers after the injury whereas the remaining 44\% of people squeeze their fingers (Figure 3). According to the protocol, we should not squeeze the finger after the injury because it increases the probability of hemolysis. In the present study, 46\% of people knew the reason whereas the other $46 \%$ of the people said it dilutes the specimen with fluid and 33\% of the people had no idea (Figure 4).

Figure 4: Pie chart represents that $21 \%$ people do not squeeze their finger because it dilutes the specimen with tissue fluid (green) and 46\% people says that it increases the probability of hemolysis (cream) and remaining 33\% of the participants had no idea (violet).

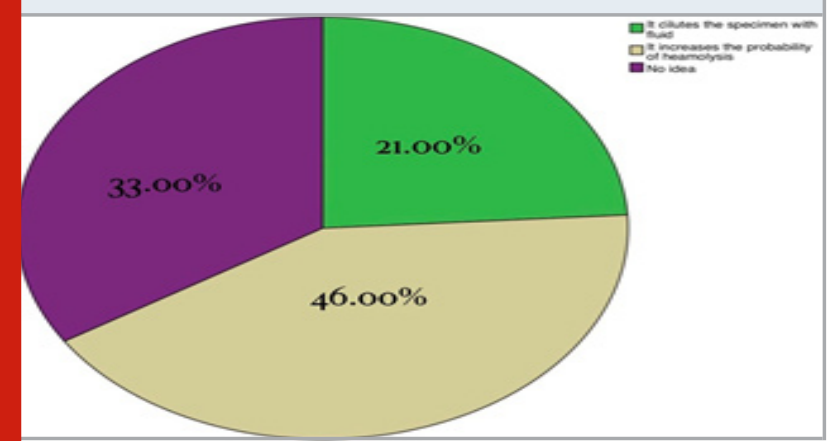

Figure 5: Pie chart represents that $46 \%$ of the participants report the incident of needle stick injury whereas the remaining (violet) 54\% of the participants do not report the incident (cream).

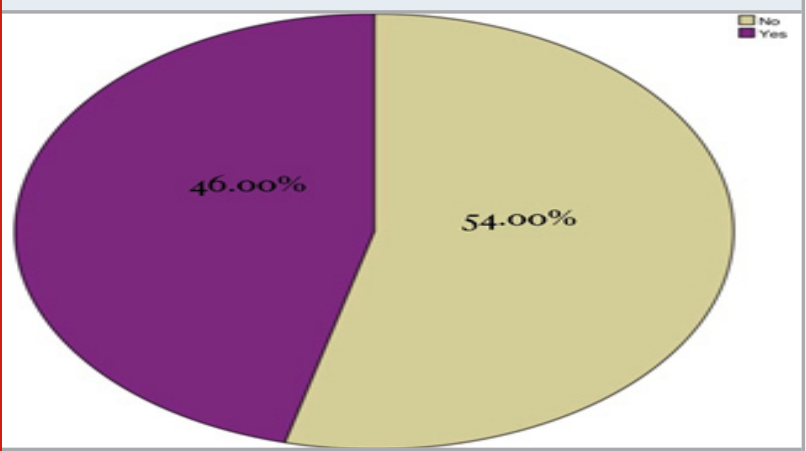

Figure 6: Pie chart represents that 35\% of the participants contact the infection control room immediately after sustaining an injury (green) and 65\% of the people immediately wash with soap after sustaining an injury (cream).

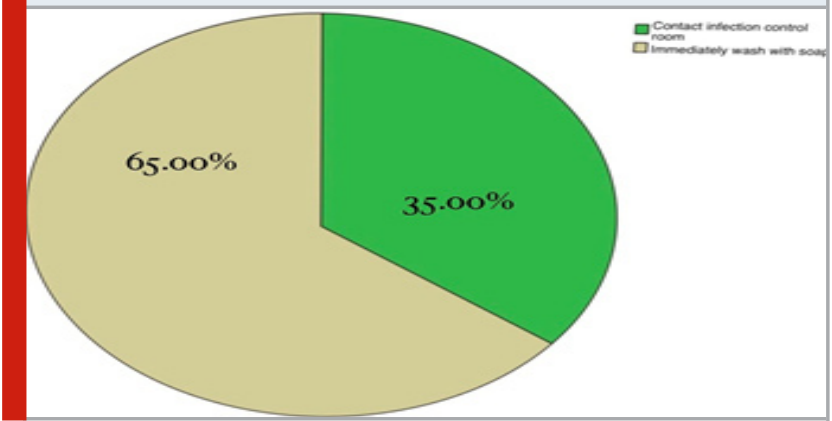


There is always confusion as to whom we should report after Needlestick injury (Salehi and Garner, 2010). According to AOTEA, medical doctors must be notified at first, who can also seek help from microbiologists and clinical immunologists for further consultations. In the present study 35\%, people contracted the infection control room immediately after the injury and the remaining 65\%people washed their hands immediately with soap (Figure 6).

The present study shows that $70 \%$ of people cap the needle before throwing it away and 30\% percent of people throw the needle before capping it (Figure 7). It also shows that $61 \%$ of people used a one-handed scoop technique whereas the remaining 39\% used two scooped hand techniques (Figure 8). Hence it can be said that people have some knowledge about needle stick injury but still, there is inadequate information.

Figure 7: Pie chart represents that 70\% of the participants cap the needle before throwing it away (cream) and 30\% of the people do not cap the needle before throwing it away (green)

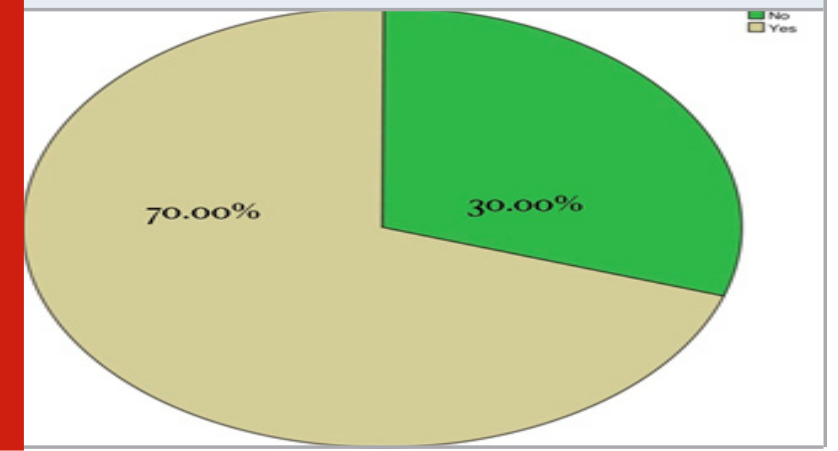

Figure 8: Pie chart represents that $61 \%$ of the participants use single handed scoop technique to cap the needle (green) and 39\% of the participants use two handed scoop technique ( cream)

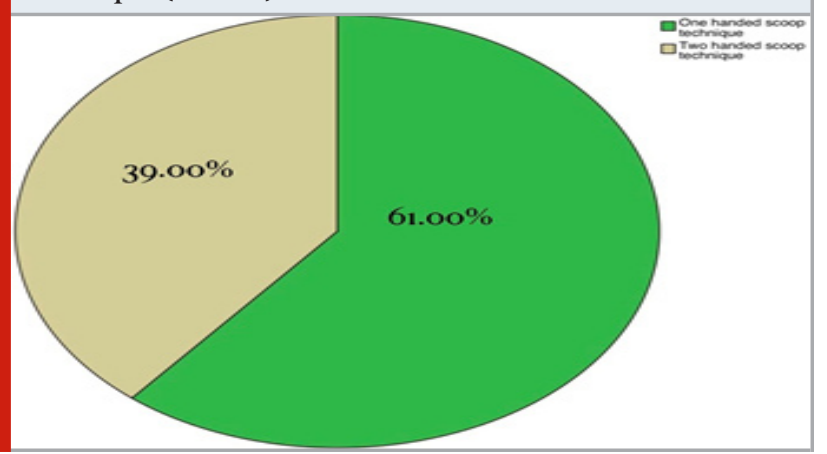

If the source person is positive for HIV, a western blot test has been done for confirmation. In this study, only $31 \%$ of people were aware of this whereas the remaining $36 \%$ and 30\% people said that HIV antibody tests and ELISA tests are done for confirmation respectively (Figure 9). Therefore it can be concluded that people should be aware of the protocol. Prevention is better than cure. The needle stick injury can also be prevented by continued education, effective training, better safety devices, and following standard precaution. In the present study 75\%, people said that students should be aware of needle stick injury during their preclinical years whereas $25 \%$ of people said that it's not necessaryl Figure 11). If the source person is negative for HBV, and if he is not vaccinated HBV vaccine is provided. In the present study 56\%, people knew that the HBV vaccine should be provided whereas the remaining 44\% of people said that the HBIG vaccine should be provided (Figure 10).

Figure 9: Pie chart represents that $31 \%$ of the participants use western blot as confirmatory test for HIV patients ( green) and 39\% of the participants use HIV antibody tests ( yellow) and 30\% of the participants use ELISA as confirmatory test (red).

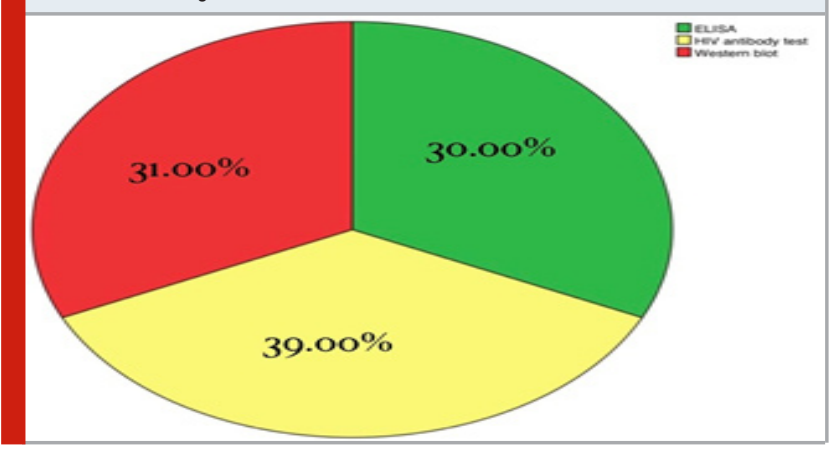

Figure 10: Pie chart represents that $44 \%$ of the participants provide HBIG vaccine to the person who has been identified as negative for HBV (green) and 56\% of the participants provide $\mathrm{HBV}$ vaccine if the affected person is negative for HBV ( cream).

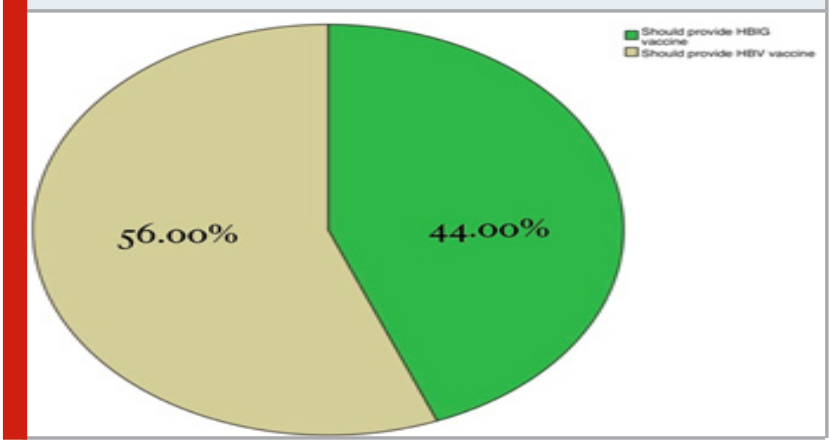

Figure 11: Pie chart represents that 75\% of the participants say that students should have knowledge about needle stick injury during their pre clinical years (cream) and $25 \%$ of the participants believe that there is no need for it (green).

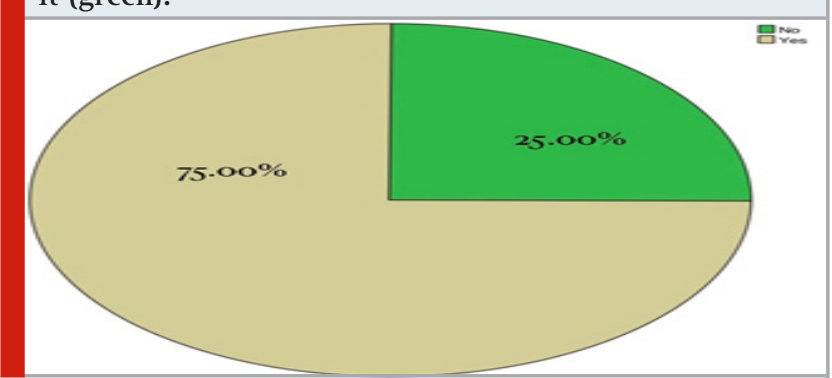


Figure 12: Bar graph represents the association between the gender and the awareness about Hepatitis B vaccine. $X$ axis represents the gender of the participants in the survey and $\mathrm{Y}$ axis represents the number of people who had been vaccinated and not vaccinated for Hepatitis B vaccine. Majority of males are vaccinated(cream) for Hepatitis $B$ than females and the difference is also significant statistically. Pearson's chi square test showing $\mathrm{p}=\mathbf{0 . 0 0}$ $(<0.05)$.

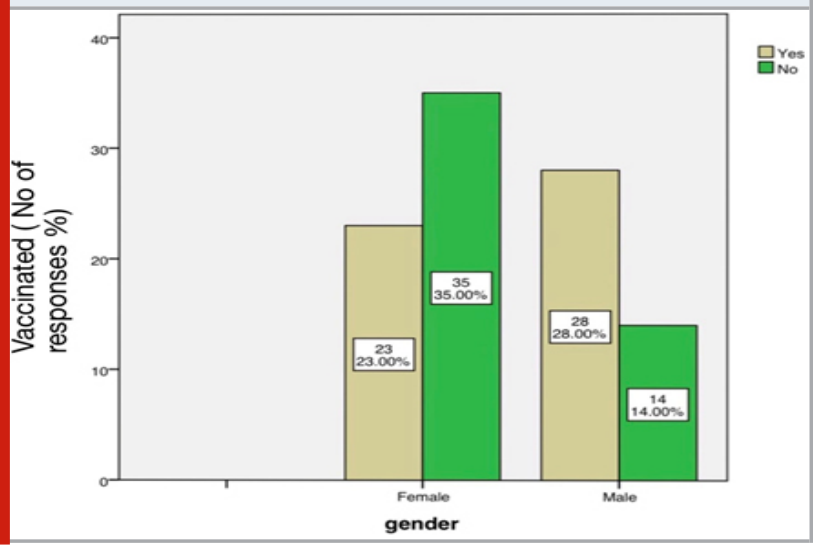

Figure 13: Bar graph represents the association between gender and checking the titre value after vaccination. $\mathrm{X}$ axis represents the gender of the participants in the survey and $\mathrm{Y}$ axis represents the number of people who check antibody titre after vaccination. Majority of the females are checked(cream) for their Hepatitis antibody titre more than the males and the difference is also significant statistically. Pearson's chi square test showing $\mathrm{p}=0.00(<0.05)$.

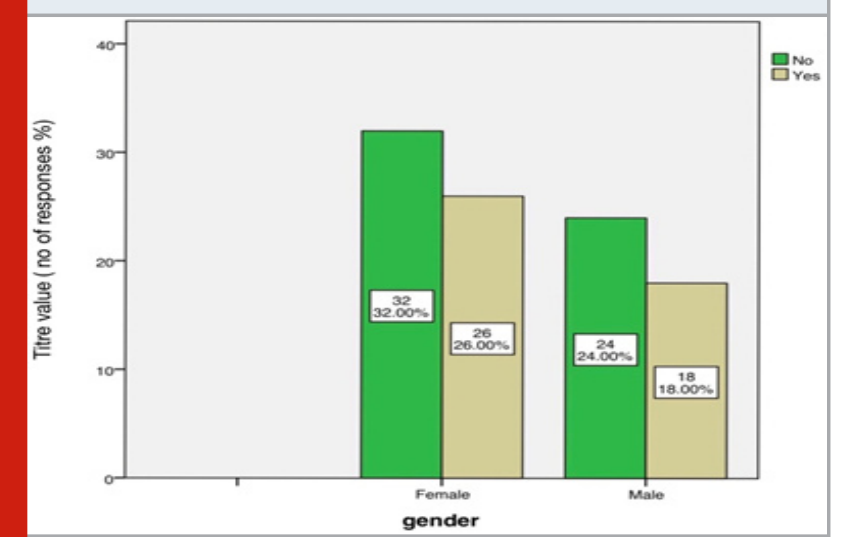

Many NSIs occur due to improper disposal of sharps e.g. unsuitable bins or on the floor and incorrect disposal of instruments by the dentists on instrumental trays(Gichki, Islam and Murad, 2015). This is reflective of a lack of knowledge and awareness among dental practitioners. An Antibody titer is used to determine whether you had previous infections and whether you need or not need certain immunization (Wick, 2001). An antibody titer test is used to measure the number of antibodies present in the blood. In the present study, only 44\% of the people checked the antibody titer after vaccination whereas the remaining 56\% of the people did not check the titer value (Figure 13). Therefore it can be said that steps that should be taken after injury are inadequate. Although a significant proportion of people knew about the infections transmitted through NSI, it was found that serological status for three major viral markers (HIV, HBV, and HCV) sources was not checked. Only 51\% of the people had been vaccinated for Hepatitis B whereas $49 \%$ of the people were not vaccinated (Figure 12).

Figure 14: Bar graph represents the association between gender and Diagnosis for Hepatitis B positive patients.X axis represents the gender and $\mathrm{Y}$ axis represents the no. of responders answered.majority of the females know that they have to check for the Hepatitis B immunisation status when they exposed to needle stick injury than the males and the difference is also significant statistically. Pearson's chi square test showing $\mathrm{p}=0.00(<0.05)$.

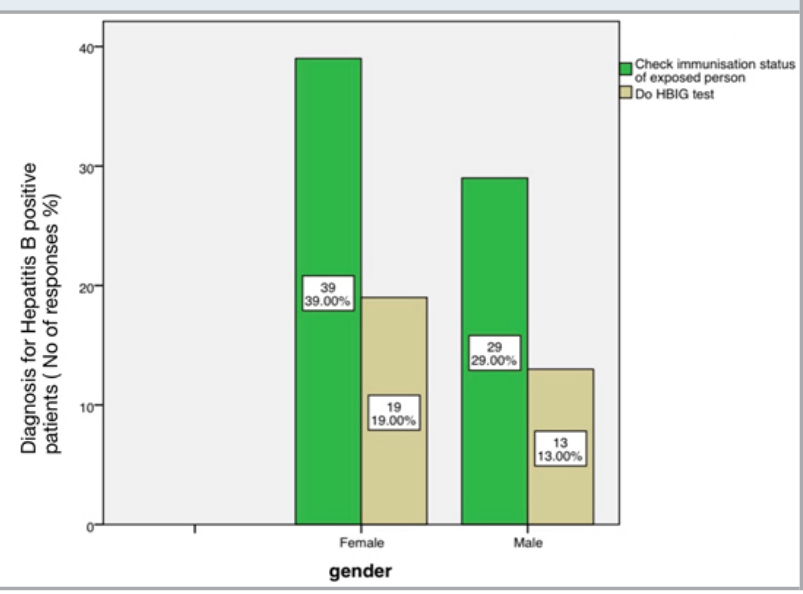

Figure 15: Bar graphs representing the association between gender and duration of HBIG vaccine have to be provided.X axis represents the gender and $\mathrm{Y}$ axis represents the no. of participants responded to the timings. Majority of females opt for 72 hours more than the males and the difference is also significant statistically. Pearson's chi square test showing $\mathrm{p}=0.00(<0.05)$.

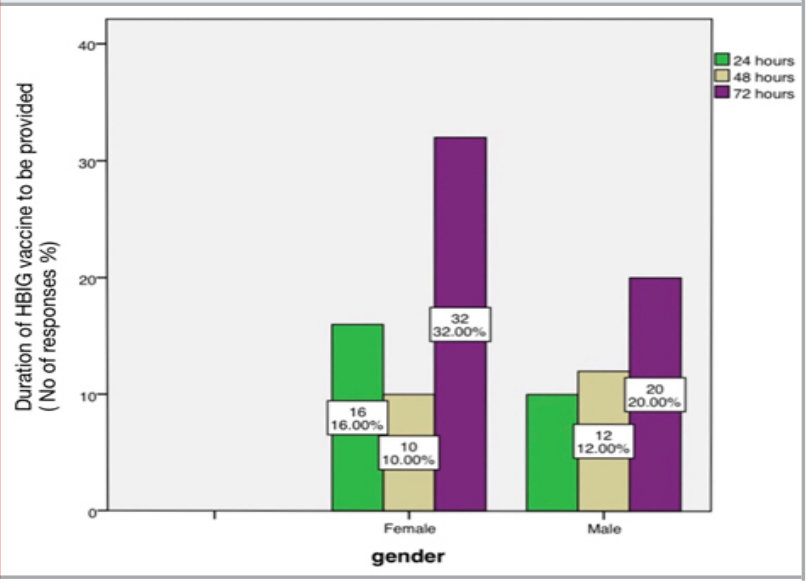

After exposure to the immunization status of the infected person should be checked and access the presence of HBV and HIV by ELISA method. If the source person is 
positive for hepatitis B, the immunization status of the person should be checked. In the present study 67\%, people were aware of this and the remaining 33\% of the people were not aware (Figure 14). If the person is positive and if he is vaccinated anti-Hbs titer should be checked. After checking the HBs titer immunoglobulin is provided within 72 hours.If the person is not vaccinated, the HBIG vaccine should be provided within 72 hours. In the present study 52\%, people were aware that HBIG should be provided within 72 hours whereas $26 \%$ of people said that it should be provided after 24 hours whereas $22 \%$ of people said that it should be provided after 48 hours (Figure 15).

Induction programs ought to be held for all recruits. The concept of regular precautions needs to be explained to all health workers and steps must be taken to put into effect them(Shah et al., 2010). Appropriate disposal of sharps and avoidance of recapping and bending should form an important thing in the training.

\section{CONCLUSION}

From this study, we conclude that only 50\% of the study population were aware about the needle stick injury and the infections spread by Needle stick injury. Even though a majority of the females knows about the Hepatitis B vaccination and its relation to needle stick injury when compared to males but they didn't undergo vaccination properly. There is a precise scope of development in terms of reporting and prevention of needlestick accidents.A need for awareness programs among dental practitioners repeatedly has been raised.

\section{REFERENCES}

Agarwal, V., Wakhlu, A. and Srivastava, P. (2016)

'Managing Needle-Stick Injury', Manual of ICU Procedures, pp. 690-690. doi: 10.5005/jp/ books/12634_62.

Alrumayyan., A. et al. (2018) 'OCCUPATIONAL HAZARD: NEEDLE STICK INJURY AMONG HEALTH CARE WORKERS', International Journal of Advanced Research, pp. 97-101. doi: 10.21474/ijar01/7649.

Ayas, N. T. et al. (2006) 'Extended Work Duration and the Risk of Self-reported Percutaneous Injuries in Interns', JAMA, p. 1055. doi: 10.1001/jama.296.9.1055.

Dafaalla, M. D. (2016) 'Knowledge, attitude and practice towards needle stick injury among health care workers in a tertiary Sudanese hospital', TEXILA
INTERNATIONAL JOURNAL OF CLINICAL RESEARCH, pp. 88-96. doi: 10.21522/tijcr.2014.03.01.art010.

Gichki, A. S., Islam, A. and Murad, W. (2015) 'KNOWLEDGE AND AWARENESS ABOUT NEEDLE STICK INJURIES AMONG DENTAL STUDENTS OF BOLAN MEDICAL COLLEGE, QUETTA', Pakistan Oral \&t Dental Journal. search.ebscohost.com, 35(4). Available at: http://search.ebscohost.com/login.aspx?direct=true \&tprofile $=$ ehost $\&$ scope $=$ siteCtauthtype $=$ crawler\&tjrnl $=1$ $0128700 \mathrm{t} A \mathrm{~N}=115820756 \mathrm{th}=\mathrm{KasEQ0X} 1 \mathrm{mt} \% 2 \mathrm{FqsX} 3 \mathrm{Y}$ dqsdh5LaN7zENmbHRI01VJn0SDsbv\%2B0JzBMEo5K V99903xiUFLVvXTvHNJyeG728x4ogEQ\%3D\%3D\&tcrl $=\mathrm{c}$.

Kapila, K., Gupta, R. M. and Chopra, G. S. (2008) 'Postexposure Prophylaxis : What Every Health Care Worker Should Know', Medical Journal Armed Forces India, pp. 250-253. doi: 10.1016/s0377-1237(08)80106-2.

Mahajan, S. and Gupta, E. (2019) 'Needle Stick Injury in Healthcare Workers', Indian Journal of Health Sciences and Care, p. 80. doi: 10.5958/23942800.2019.00016.6.

Nanda, L. et al. (2019) 'Effectiveness of Structured Teaching Program regarding Prevention of Needle Stick Injury among Nursing Students', Indian Journal of Holistic Nursing, pp. 27-30. doi: 10.24321/2348.2133.201904. Olubuyide, I. O. and Olawuyi, F. (1995) 'Self-reported incidence of accidental exposures to patients' blood and body fluids by resident doctors in Nigeria', Journal of the Royal Society of Health, pp. 235-243. doi: $10.1177 / 146642409511500408$.

Shah, R. et al. (2010) 'Knowledge and awareness regarding needle stick injuries among health care workers in tertiary care hospital in Ahmedabad, Gujarat', Nat J Com Med. njcmindia.org, 1(2), pp. 93-96.

Singh, D. A. et al. (2017) 'Needle Stick Injuries among Healthcare Waste Handlers in a Tertiary Care Hospital of Delhi', Epidemiology International, pp. 14-17. doi: 10.24321/2455.7048.201706.

Walley, S. (2014) 'Reporting occupational exposure to blood and body fluids', Dental Nursing, pp. 608-609. doi: 10.12968/denn.2014.10.10.608.

Wick, J. (2001) 'New Standards To Prevent Needlestick Injury', Journal of Managed Care Pharmacy, pp. 349-352. doi: 10.18553/jmcp.2001.7.5.349. 